\title{
On the fusion of iridium and rhodium
}

\section{R. Hare}

To cite this article: R. Hare (1847) On the fusion of iridium and rhodium, Philosophical Magazine Series 3, 31:206, 147-150, DOI: 10.1080/14786444708645807

To link to this article: http://dx.doi.org/10.1080/14786444708645807

\section{曲 Published online: 30 Apr 2009.}

Submit your article to this journal

Џ Article views: 2

Q View related articles ¿ 
water-bath until the sulphur is seen to be no longer altered and the liquid has assumed a yellow colour; it is then heated to boiling, and kept at this temperature until the sulphuret of ammonium has been expelled and the liquid has again become colourless. The deposited, or excess of, sulphur is now removed by filtration, and the liquid evaporated to crystallization. In this way from $3 \frac{1}{3}$ to $3 \frac{1}{2} \mathrm{oz}$. of dazzling white dry sulphocyanide of ammonium are obtained, which may be employed as a reagent, and for the same purposes as the sulphocyanide of potassium. Of the $2 \mathrm{oz}$. of sulphur added, $\frac{1}{2}$ an $0 \mathrm{z}$. is left undissolved.

The behaviour of the higher sulphurets of ammonium towards prussic acid furnishes an admirable test for this acid. A couple of drops of a prussic acid, which has been diluted with so much water that it no longer gives any certain reaction with salts of iron by the formation of prussian blue, when mixed with a drop of sulphuret of ammonium and heated upon a watch-glass until the mixture is become colourless, yields a liquid containing sulphocyanide of ammonium, which produces with persalts of iron a very deep blood-red colour, and with persalts of copper, in the presence of sulphurous acid, a perceptible white precipitate of the sulphocyanide of copper. -Liebig's Annalen, Jan. 1847.

\section{ON THE FUSION OF IRIDIUM AND RHODIUM. BY R, HARE.}

This communication respects mainly my success in fusing both iridium and rhodium, neither of which, in a state of purity, had been previously fused. It may be supposed that the globule of iridium, obtained by Children's colossal battery, forms an exception; but the low specific gravity and porosity of that globule may justify a belief that it was not pure, and at any rate the means employed were of a nature not to be at command for the repetition of the process, so that iridium might as well be infusible, as to be fusible only by such a battery.

The first specimen of the last-mentioned metal on which I operated was one given me by Mr. Booth, a former pupil of Wöhler, whom he had assisted in obtaining it by the excellent process devised by that distinguished chemist. This specimen was fused in the presence of Mr. Booth. Subsequently I procured specimens, warranted pure, severally from the house of Pelletier at $\mathbf{P}$ aris, and from Messrs. Johnson and Cock, London. Another specimen was given to me by a friend, who had received it as pure, from a source on which reliance may be placed; and lastly, I obtained myself, by Wöhler's process, a specimen of about sixty grains, from the insoluble resiudue of platinum ore. All the specimens thus procured were found to be fusible under my hydro-oxygen blowpipe. The specimen obtained from Messrs, Johnson and Cock, after repeated fusions, by which it was much consolidated, weighed sixty-seven grains. During fusion there appeared to be an escape of volatile matter, supposed to be osmic acid, arising from the presence of a minute portion of osmium, between which and iridium an affinity of 
a peculiar degree of energy exists. At a certain point of the process a reaction took place sufficiently explosive to throw a portion of the metal, in globules, off from the support. One of these, about twice as large as the head of a common brass pin, proved to be hollow. By prolonged and repeated fusion the metal became more compact and more fusible.

Fused iridium has nearly the grain of soft cast steel, with the pale whiteness of antimony, and appears to be susceptible of a fine polish. Although as hard as untempered steel, it is somewhat sectile, since, when split by means of a cold chisel, the edge penetrated about the eighth of an inch before a division was effected. By light hammering a corner was flattened without fracture, although under heavier blows the mass cracked. I infer that although nearly unmalleable and very hard, iridium may be wrought in the lathe.

I have already mentioned that I fused into a globule a specimen of iridium obtained by me from the insoluble residuum of platinum ore by Wöhler's process. From this globule, while congealing, a portion ran out from the inside, leaving a cavity and covering one of its sides externally with an incrustation, among which crystalline spangles, or facets, were discernible. The specific gravity of the globule of iridium, from the specimen furnished by Messrs. John. son and Cock, was taken by Mr. T. R. Eckfelt of the United States mint at Philadelphia, and by Dr. Boyé, both having balances of the greatest accuracy, and being very skilful in the employment of them. In the first instance there was a perfect coincidence in the results obtained, 21.83 being the numbers found by both of these gentlemen. Agreeably to another trial made by Dr. Boyé, using river-water instead of distilled, the number was $21 \cdot 78$, water being in either case about sixty-eight, with allowance for the difference of the water, and the temperature being above the standard of $60^{\circ}$. The specific gravity of the specimen may then be estimated at $21 \cdot 80$.

The specific gravity of fused platinum, purified according to the instructions of Berzelius, before subjection to the hammer, proved in one specimen to be not more than $19 \cdot 70$, although by hammering it became equal to 21.23 . It is with fused platinum that fused iridium should be compared. Of course the specific gravity of the last-mentioned metal, when both are obtained by fusion, may be assumed to be one-tenth greater than that of the former. Moreover, as this metal is the only impurity existing in the standard platinum of London, of Paris, or of St. Petersburg, it follows that a high specific gravity is not to be viewed as a proof of purity. Accordingly a specimen of platinum, purified from iridium by the Berzelian process, and which had proved eminently susceptible of being beaten into leaf, was found only to be of the gravity of $21 \cdot 16$, while that of a specimen of standard Russian platinum, very brilliantly white but inferior in malleability, presented to me by his Excellency Count Cancrine, as a specimen of the purest platinum of the Russian mint, was $21 \cdot 31$.

Of rhodium I have fused two specimens, one of five pennyweights, purchased of Messrs. Johnson and Cock, the other received through 
the same channel as the specimen of iridium above-mentioned*. Rhodium is at least as fusible as iridium, both of the specimens alluded to having been converted into fluid globules. That procured from Messrs. Johnson and Cock gave a globule weighing ninety grains. On a second fusion it formed a perfect globule as fluid as mercury; and yet in congealing lost its brilliancy by becoming studded with crystalline facets all over its surface, excepting the portion in contact with the support. The facets had the appearance of incipient spangles. The rapidity with which they were formed seemed anomalous. The mass being split by a cold chisel and viewed by a microscope, it appeared porous immediately beneath the facets. When the mass was first fused, I found by the gravimeter the specific gravity to be $11 \cdot 0$, which coincides with the observations of Wollaston. Yet by a careful trial made at the United States mint by Mr. Eckfelt, after the second fusion and the formation of the facet, the specific gravity proved to be only $10 \cdot 8$. This is sufficiently explained by the porosity above mentioned. In fact the porosity to which rhodium and iridium are liable may render it difficult to find specimens of precisely the same specific gravity.

In sectility, malleability and hardness, rhodium did not appear to differ much from iridium, but it is not of so pale a white as iridium. The one has the pale white of antimony, the other the ruddy hue of bismuth.

Osmiuret of iridium, as existing in the native spangles associated with platina ore, or as otherwise obtained, is far more difficult of fusion than pure iridium. The propensity to assume the crystalline form, and to adhere to it, is even greater in this alloy than in the last-mentioned metal. On first exposure to the most intense heat of the lydro-oxygen blowpipe some slight appearances of fusion may be seen, and the spangles or grains may be made to cohere. Nevertheless it yields very slowly, and requires an expenditure of gas too great to be incurred unless it were for the purpose of once well determining the question of its ultimate fusibility. This object was obtained completely as respects a globule of 45 grains in weight. The specific gravity of this globule appeared to be 20.4 , but this result was evidently less than that which would have been obtained had there not been some minute cavities, which, after splitting the globule, were detected by a magnifier.

The specific gravity of some large spangles of osiniuret of iridium from South American ore was, by Dr. Boyé, found to be $19 \cdot 835$. That of sume grains heavier but not so flat, presented to me by Count Cancrine, was found to be 20.938.

That the alloy of iridium with osmium should be more difficult to fuse than pure iridium, leads to the inference that osmium must be the most infusible of the metals, although, like carbon, very susceptible of combustion, and capable, like that infusible non-metallic radical, of forming a volatile peroxide. Of course its liability to oxidizement would render it impossible to fuse it by the hydro-

* One other larger specimen from the same source has been fused since the above was written. 
oxygen blowpipe, of which the efficacy requires the simultaneous presence of oxygen and the most intense heat. It might be fused by exposure in vacuo to the discharge of a powerful voltaic series, by means of the apparatus of which a description with engravings has been given in a recent volume of the Transactions of the American Philosophical Society, and republished in 'Silliman's Journal' for 1841 , vol. xl. p. 303.

I have obtained osmium by heating the osmiate of ammonia in a glass tube with sal-ammoniac, agreeably to the instructions given by Berzelius. In this way a result was obtained which the information given by that distinguished chemist had not led me to anticipate. The tube became coated with a ring of osmium, which it would be impossible by inspection merely to distinguish from the arsenical ring on the peculiar features of which reliance has been placed for the detection of arsenic.

It follows from my experiments and observations, that of all metallic bodies, osmiuret of iridium is the most difficult to fuse; that rhodium and iridium are both fusible by the hydro-oxygen blowpipe, properly employed; that the former has the rosy whiteness of bismuth, the latter the pale white of antimony; and that both of them are slightly sectile, though extremely hard and nearly unmalleable; that iridium merely fused is heavier than platinum condensed by the hammer. Thus it follows from my experiments, and from the recent observations of Breithaupt, on some specimens of native iridium, that the metal, whether in this state or pure as obtained by chemical skill and consolidated by fusion, must be allowed that pre-eminence in density, which, until of late, was given to platinum.

It may be proper to add, that subsequently to the writing of the preceding narrative, receiving some large quantities of iridium and rhodium from Messrs. Johnson and Cock, my experiments were successfully repeated on a larger scale, but without any result besides that of confirming the facts above stated.-Silliman's Journal for Nov. 1846, p. 365 .

NOTE ON THE MEANS OF TESTING THE COMPARATIVE VALUE OF ASTRINGENT SUBSTANCES FOR THE PURPOSES OF TANNING. BY ROBERT WARINGTON, ESQ.

Having been frequently called upon to examine the value of astringent substances imported into this country for the purposes of tanning, such as valonia, divi-divi, sumac, cutch, \&c., I am induced to believe that the detail of the manipulation adopted may not be without interest to some of the members of the Society, As the manufacture of leather was the object of the purchaser of these materials, gelatin was selected as the basis for the estimation of their comparative value; and after several trials with various kinds of natural and manufactured gelatin, such as varieties of isinglass, glue, patent gelatin, \&c., the finest long staple isinglass was found to be the most constant in its quality and least liable to undergo change. 